\title{
Pilot Scale Extraction of Orthosiphon stamineus Raw Material as Rosmarinic Acid Extract
}

\author{
Cher Haan Lau ${ }^{1,2}$ and Lee Suan Chua ${ }^{1,2, *(D)}$ \\ 1 Department of Bioprocess and Polymer Engineering, School of Chemical and Energy engineering, \\ Faculty of Engineering, Universiti Teknologi Malaysia, 81310 UTM Skudai, Johor Bahru, Johor, Malaysia; \\ cherhaan@gmail.com \\ 2 Institute of Bioproduct Development, Universiti Teknologi Malaysia, 81310 UTM Skudai, Johor Bahru, \\ Johor, Malaysia \\ * Correspondence: chualeesuan@utm.my; Tel.: +60-197214378
}

Received: 5 January 2019; Accepted: 26 February 2019; Published: 27 February 2019

\begin{abstract}
A scale-up protocol for rosmarinic acid extraction from Orthosiphon stamineus was developed using the concept of constant power dissipation for a similar kinetic profile. Rosmarinic acid is the bioactive compound in the herb and therefore, large scale extraction for that compound is important for commercial application. The first-order kinetic equation which was established in the preparative scale extraction was used to describe the extraction of rosmarinic acid from the herb. The agitation speed of the pilot large-scale extractor ( $451 \mathrm{rpm})$ was determined based on the constant power dissipation to meet the minimal required speed, namely just-suspended speed $\left(N_{j s}\right)$, $450.84 \pm 0.88 \mathrm{rpm}$. The experimental data was fitted well to the proposed kinetic equation with a high correlation coefficient, $R^{2} 0.88$, and a low root mean square error, RMSE 3.85. This indicates the good performance of the scale-up approach based on the dynamic criterion. Therefore, the use of constant power dissipation to obtain a similar kinetic profile could be applied for phytochemical extraction from herbal plants.
\end{abstract}

Keywords: extraction; scale-up; kinetic model; Orthosiphon stamineus; rosmarinic acid; power dissipation

\section{Introduction}

The establishment of a herbal processing platform, particularly for large-scale production, is very important to fulfill the increasing demand for herbal products [1]. The most important criterion for a large-scale processing platform is the capacity to produce a high yield of phytochemicals with consistent quality. The quality of the herbal extract is usually subjected to the presence of a marker compound. It is difficult to produce a herbal extract with a high consistency of quality and quantity for large-scale production [2].

Scaling up of the herbal processing technology is challenging because it is difficult to control the output of the highly complex system. Most of the scale-up processes were carried out by a trial-and-error method [3]. The scale-up procedure also varies according to the type of reactor and process conditions, as well as plant origin. Even though many studies have been carried out for Orthosiphon stamineus extraction, the published technical data on pilot-scale or large-scale processes are relatively limited in literature. The herbal extraction is often scaled up in terms of the geometric aspect of the vessel rather than the dynamic aspect [4]. The dynamic aspect is believed to be more reliable in achieving a successful scale-up process. Therefore, this study presents the works on scaling up rosmarinic acid extraction from $O$. stamineus using the dynamic concept. 


\section{Materials and Methods}

\subsection{Plant Material}

The raw materials of O. stamineus (white flower species) were supplied by Fidea Resources (Selangor, Malaysia). The stems and leaves of the plant were dried and ground into a fine powder $(<1 \mathrm{~mm})$ before extraction. The specimen of the fresh plant was authenticated by Forest Research Institute Malaysia (Kuala Lumpur, Malaysia), reference number: SBID 001/13.

\subsection{Preparative Scale and Pilot Scale Reflux Extraction}

The preparative scale extraction was carried out using the extraction conditions as reported in previous studies [5]. The plant samples $(273.5 \mathrm{~g})$ were extracted for $3 \mathrm{~h}$ with $70 \%$ ethanol $(2500 \mathrm{~mL})$ at $60{ }^{\circ} \mathrm{C}$. The kinetic model of $O$. stamineus extraction was developed based on the experimental data of a preparative-scale extraction process. The first-order kinetic model is presented in Equation (1). A $10 \mathrm{~mL}$ sample was withdrawn at $15 \mathrm{~min}$ intervals for rosmarinic acid analysis during extraction.

$$
C_{t}=37.345\left(1-e^{-0.302 t}\right)
$$

The pilot scale extraction was conducted using a $13 \mathrm{~L}$ water-jacketed borosilicate reactor (Labfors 5, INFORS HT, Bottmingen, Switzerland), equipped with three 6-blade Rushton impellers and a temperature sensor. The temperature was controlled by a thermostatic bath/circulator (LAUDA Alpha RA 8, LAUDA-Brinkmann LP, NJ, USA). Likewise, a $10 \mathrm{~mL}$ sample was withdrawn at 15 min intervals for $3 \mathrm{~h}$ for rosmarinic acid analysis.

\subsection{Quantification of Rosmarinic Acid by HPLC-DAD}

The concentration of rosmarinic acid was determined by using a liquid chromatography system (Agilent 1260 Infinity LC, Agilent Technologies Inc., Palo Alto, CA, USA), equipped with a photodiode array detector, an auto-sampler, and a reversed-phase C18 column (Zorbax Eclipse Plus, $4.6 \times 100 \mathrm{~mm}$, $3.5 \mu \mathrm{M}$, Agilent Technologies Inc., Palo Alto, CA, USA). The column temperature was maintained at ambient temperature and the mobile phase consisted of $0.1 \%$ formic acid in water (A) and acetonitrile (B). The separation was performed in a gradient elution with the following proportions (v/v) of solvent A: 0-5 min, 85\%; 5-10 $\mathrm{min}, 85-40 \%$; 10-18 $\mathrm{min}, 40 \%$; 18-25 $\mathrm{min}, 40-85 \%$; and 25-30 $\mathrm{min}, 85 \%$, at a flow rate of $0.75 \mathrm{~mL} / \mathrm{min}$. The wavelength of the detector was set at $254 \mathrm{~nm}$. The standard solution was prepared by dissolving $5 \mathrm{mg}$ of standard chemicals (eupatorine, sinensetin, $3^{\prime}$-hydroxy-5,6,7,4'-tetramethoxyflavone, and rosmarinic acid) in $5 \mathrm{~mL}$ of ethanol-water (7:3). The stock standard solution was diluted with different dilution factors to prepare a serial concentration of standard solutions (20-100 ppm). The solutions were filtered by nylon membrane filters $(0.45 \mu \mathrm{M})$ before HPLC injection.

\subsection{Scaling up Rosmarinic Acid Extraction}

The scale-up process was carried out based on the similarity theory to obtain the similar kinetic profile as in the preparative-scale extraction under similar extraction conditions. Some assumptions for the scale-up procedures are as below:

i. The extraction was an isothermal and isobaric process;

ii. The preparative- and pilot-scale extraction vessels were geometrically similar;

iii. The stirred vessel system was fully turbulent.

The scale-up protocol was modified from the procedures suggested by Alam et al. [6], who highlighted the application of the similar rate constant $(k)$ in both preparative- and pilot-scale systems. The overall flow of the scale-up process is presented in Figure 1. The agitation speed of the pilot-scale system was also fixed at the constant power dissipation (power per unit volume) as the value for the 
preparative-scale extractor. The complete suspension in the stirred tank can be measured using the Zwietering's correlation, as presented in Equation (2), which is used to predict just-suspended speed, $N_{j s}$ [7]. Table 1 lists the properties of preparative- and pilot-scale reactors.

$$
N_{j s}=\frac{S\left(g \Delta \rho / \rho_{l}\right)^{0.45} d_{p}^{0.2} X^{0.13} v^{0.1}}{D^{0.85}}
$$

where $S$ is the Zwietering's constant, $g$ is the gravitational constant $\left(9.81 \mathrm{~m} / \mathrm{s}^{2}\right), \Delta \rho$ is the density difference between solid and liquid, $\rho_{l}$ is the density of the liquid $\left(\mathrm{kg} / \mathrm{m}^{3}\right), \mathrm{d}_{\mathrm{p}}$ is the solid diameter $(\mathrm{m}), \mathrm{X}$ is the solid concentration (mass ratio of solid), $v$ is the kinematic viscosity of liquid $\left(\mathrm{m}^{2} / \mathrm{s}\right)$, and $\mathrm{D}$ is the impeller diameter $(\mathrm{m})$.

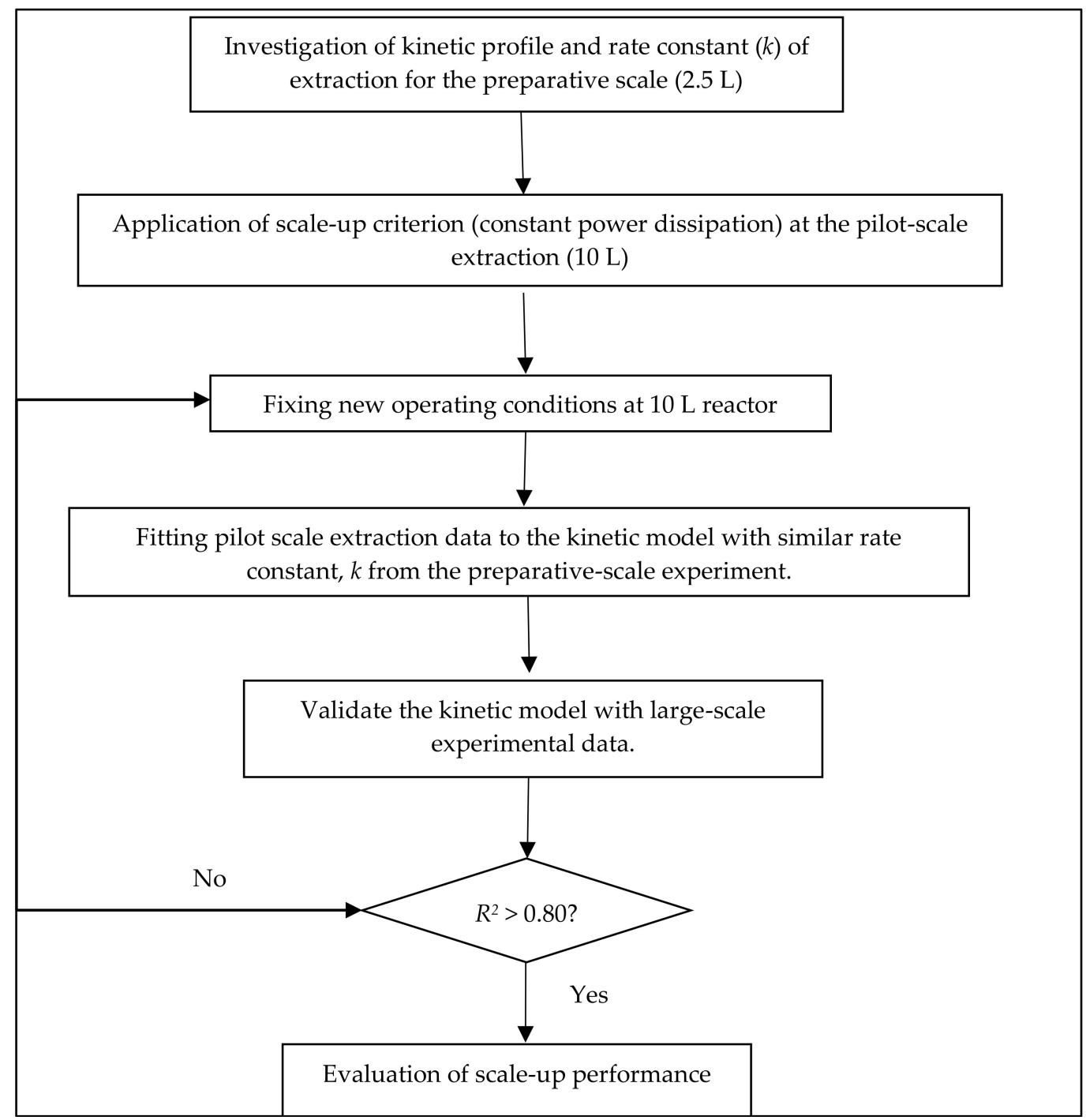

Figure 1. Scale-up protocol based on the similarity in the kinetic profile. 
Table 1. Properties of preparative- and large-scale reactors.

\begin{tabular}{ccc}
\hline Properties & Preparative-Scale Extractor & Pilot-Scale Extractor \\
\hline Vessel volume, $\mathrm{V}_{\text {vessel }}\left(\mathrm{m}^{3}\right)$ & 0.005 & 0.013 \\
Working volume, $\mathrm{V}_{\text {working }}\left(\mathrm{m}^{3}\right)$ & 0.0025 & 0.01 \\
Vessel diameter, $\mathrm{T}(\mathrm{m})$ & 0.18 & 0.2 \\
Liquid level, $\mathrm{z}(\mathrm{m})$ & 0.0918 & 0.3183 \\
Impeller diameter, $\mathrm{D}(\mathrm{m})$ & 0.06 & 0.07 \\
Liquid density, $\rho \mathrm{L}\left(\mathrm{kg} / \mathrm{m}^{3}\right)$ & & \\
Liquid viscosity, $\mu(\mathrm{Pa} \cdot \mathrm{s})$ & & \\
\hline Scale-up with constant power dissipation $(\mathrm{P} / \mathrm{V})$ & 0.002225 & \\
\hline Agitation speed, $\mathrm{N}(\mathrm{rpm})$ & 500 & 14,54 \\
Reynolds number, Re & $11,849.5$ & (Fully turbulent) \\
Top speed, $\pi \mathrm{ND}$ & (Fully turbulent) & 1.65 \\
\hline
\end{tabular}

\section{Results and Discussion}

The extraction process of a herbal material is a solid-liquid operation which involves solid suspension (also known as coarse dispersion) and dispersion. The raw material is suspended in liquid (medium) and the active compounds (phytochemicals) are dispersed in medium. According to Geisler et al. [8], the desired state of solid suspension is essential for successful reactor design and process scale-up. The solid particles of herbal material shall be distributed within the entire stirred liquid during extraction. In other words, a complete suspension (no solid settlement on the vessel bottom) must be achieved during the process. The active compounds extracted from the herbal material shall also form a homogenous suspension. Therefore, a constant power input or power consumption is considered to be more reliable as a scale-up criterion for rosmarinic acid extraction from a highly complex phytochemical mixture of the herbal plant [8].

The scale-up process mostly affected the hydrodynamic characteristics, thus reducing the performance of the larger scale system [9]. This included affecting the mass transfer of phytochemicals because of the increment in the radial non-uniformity of velocity in the larger system [10]. Therefore, the scaled-up extractor tended to form stagnation zones in the stirred tank and hinder the complete suspension state of the system [11]. In the present study, the $N_{j s}$ was $450.84 \mathrm{rpm}$, which is almost similar to the agitation speed calculated by the constant $\mathrm{P} / \mathrm{V}, 451 \mathrm{rpm}$. This indicates that the impeller speed met the minimum requirement to achieve complete suspension. $N_{j s}$ is the minimum agitation speed which is required to suspend solid particles so that they are homogenously dispersed in the medium. $N_{j s}$ is also considered to be the optimal operating point for effective mass transfer of rosmarinic acid in the vessel. Hence, the agitation speed of the vessel must meet the $N_{j s}$ in order to achieve complete suspension [7]. The detailed calculations of $\mathrm{P} / \mathrm{V}$ and $N_{j s}$ are provided in the Supplementary Materials (Appendix A).

The kinetic data of the pilot-scale extraction was fitted into the kinetic model developed in the preparative scale as presented in Equation (1). The performance of both preparative- and pilot-scale extractors was also compared in terms of their kinetic profiles. It was found that the experimental data of pilot-scale extraction was fitted well to the kinetic equation with the correlation coefficient $R^{2} 0.88$ and the root mean square error RMSE 3.85. Figure 2 illustrates the kinetic profile of rosmarinic acid extraction in the preparative- and pilot-scale extractors. Both extractors had almost a similar kinetic profile. The figure also clearly indicates that the concentration of rosmarinic acid extracted from the plant material was in line with the calculated values using the first-order kinetic equation, as indicated by the solid line. The chromatogram of $O$. stamineus extract is presented in Figure 3. Rosmarinic acid is the highest peak detected at $11.5 \mathrm{~min}$. The other compounds included $3^{\prime}$-hydroxy-5,6,7,4'-tetramethoxyflavone, sinensetin, and eupatorine. The findings revealed that fixing constant power dissipation could be a good scale-up criterion for rosmarinic acid extraction in a stirred 
tank reflux system. This dynamic parameter had been successfully used to scale up the process with almost a similar kinetic profile. The chromatograms of preparative- and pilot-scale processes are presented in Supplementary Figure S1 (Supplementary Materials).

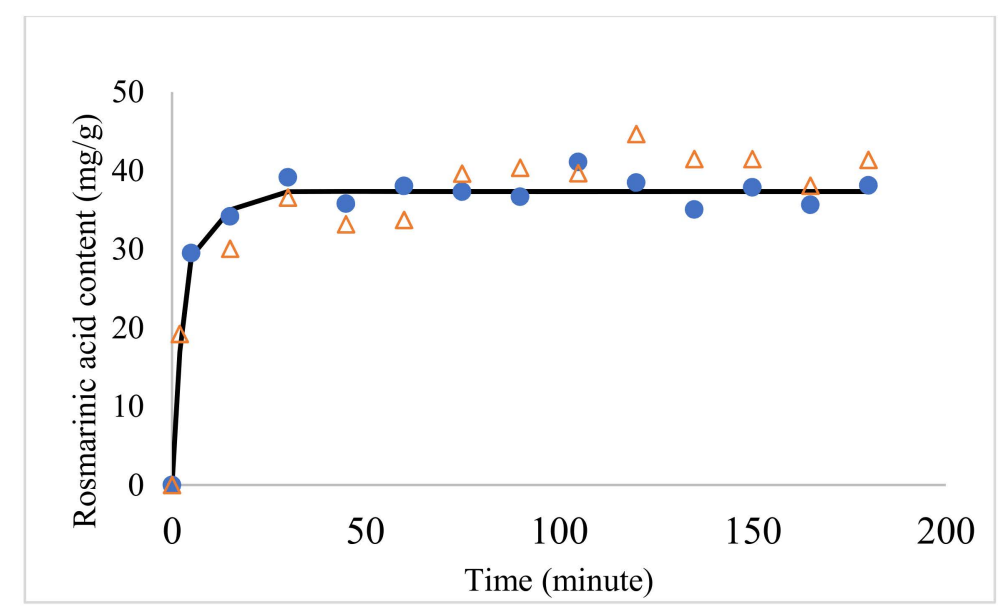

Figure 2. Kinetic profile of rosmarinic acid extraction in preparative- $(\bullet)$ and pilot-scale processes $(\Delta)$ plotted with the predicted first-order kinetic model (line).

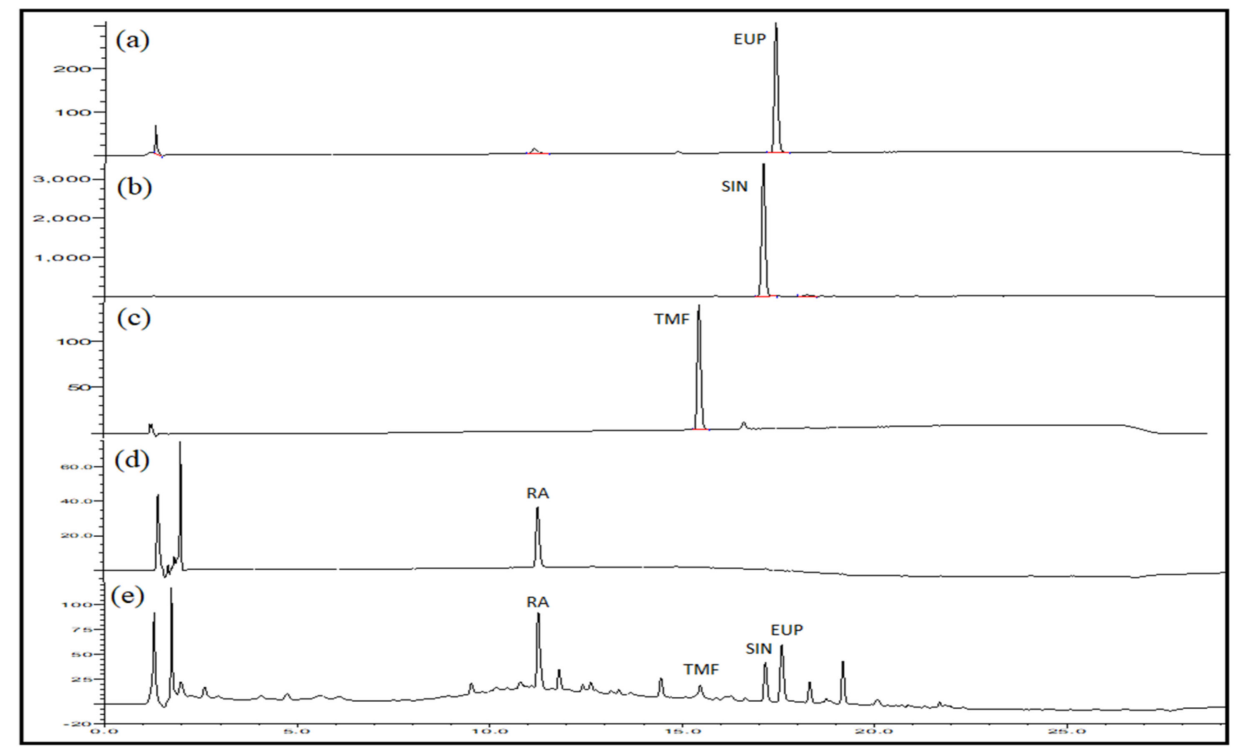

Figure 3. Chromatograms of (a) eupatorin (EUP), (b) sinensetin (SIN), (c) 3'-hydroxy-5,6,7,4'-tetramethoxyflavone (TMF), (d) rosmarinic acid (RA), and (e) Orthosiphon stamineus extract.

\section{Conclusions}

The reflux extraction of rosmarinic acid from the stems and leaves of $O$. stamineus was successfully scaled up to pilot scale $(10 \mathrm{~L})$ based on the constant power dissipation. The first-order kinetic equation was proved to describe the extraction curve of rosmarinic acid in both preparative and pilot scales. In addition, the dynamic criterion to keep constant power dissipation was reliably applied in the scaling-up process of rosmarinic acid extraction. The kinetic model and scale-up performance of rosmarinic acid extraction from O. stamineus provide important information for the herbal processing industry.

Supplementary Materials: The following is available online at http://www.mdpi.com/2624-7402/1/1/7/s1. 
Author Contributions: Conceptualization, L.S.C.; methodology, L.S.C.; validation, C.H.L.; data curation, C.H.L.; writing-original draft preparation, C.H.L.; writing—review and editing, L.S.C.; supervision, L.S.C.; project administration, L.S.C.; funding acquisition, L.S.C.

Funding: This research was funded by Ministry of Higher Education, Malaysia, 4J263.

Acknowledgments: The authors would like to thank the department technicians to assist in operating the pilot-scale extractor.

Conflicts of Interest: The authors declare no conflict of interest.

\section{References}

1. Vasisht, K.; Kumar, V. Trade and Production of Herbal Medicines and Natural Health Products; United Nations Industrial Development Organization (UNIDO) and the International Centre for Science and High Technology (ICS), Earth, Environmental and Marine Sciences and Technologies: Trieste, Italy, 2002.

2. Gaedcke, F. Quality assurance of herbal medicinal products. In Herbal Medicinal Products, Scientific and Regulatory Basis for Development Quality Assurance Marketing Authorization; Gaedcke, F., Steinhoff, B., Blasius, H., Eds.; Medpharm Scientific Publishers and CRC Press: Stutgatt, Germany, 2003; Chapter 3, pp. 37-63.

3. Mohammad Azmin, S.N.H.; Abdul Manan, Z.; Wan Alwi, S.R.; Chua, L.S.; Mustaffa, A.A.; Yunus, N.A. Herbal processing and extraction technologies. Sep. Purif. Rev. 2016, 45, 305-320. [CrossRef]

4. Raval, N.; Tambe, V.; Maheshwari, R.; Deb, P.K.; Tekade, R.K. Scale-up studies in pharmaceutical products development. In Dosage Form Design Consideration, Advances in Pharmaceutical Product Development and Research; Tekade, R.K., Ed.; Academic Press, Elsevier Inc.: Massachusetts, USA, 2018; Volume 1, Chapter 19; pp. 669-700.

5. Lau, C.H.; Chua, L.S.; Lee, C.T.; Aziz, R. Optimization and kinetic modeling of rosmarinic acid extraction from Orthosiphon stamineus. Curr. Bioact. Compd. 2014, 10, 271-285. [CrossRef]

6. Alam, Z.; Hisham, M.N.; Razali, F. Scale-up of stirred and aerated bioengineering bioreactor based on constant mass transfer coefficient. J. Teknol. 2005, 2005, 95-110.

7. Brown, D.A.R.; Jones, P.N.; Middleton, J.C.; Papadopoulos, G.; Arik, E.B. Experimental Methods, Handbook of Industrial Mixing; John Wiley \& Sons, Inc.: Hoboken, NY, USA, 2004; pp. 145-256.

8. Geisler, R.K.; Buurman, C.; Mersmann, A.B. Scale-up of the necessary power input in stirred vessels with suspensions. Chem. Eng. J. 1993, 51, 29-39. [CrossRef]

9. Rozen, A.M.; Kostanyan, A.E. Scaling-up effect in chemical engineering. Theor. Found. Chem. Eng. 2002, 36, 307-313. [CrossRef]

10. Boyadjiev, C. Quantitative Analysis, Theoretical Chemical Engineering: Modeling and Simulation; Springer: Berlin/Heidelberg, Germany, 2010; pp. 243-296.

11. Atiemo-Obeng, V.A.; Penney, W.R.; Armenante, P. Solid-Liquid Mixing. In Handbook of Industrial Mixing: Science and Practice; Paul, E.L., Atiemo-Obeng, V.A., Kresta, S.M., Eds.; John Wiley \& Sons, Inc.: New York, NY, USA, 2004; pp. 543-584. 